\title{
FACHADAS VIDRIADAS ESTRUCTURADAS EN MADERA
}

\author{
CRISTIÁN SCHMITT | UNIVERSITY OF BRITISH COLUMBIA
}

Arquitecto con Certificado Académico en Física Fundamental, Pontificia Universidad Católica de Chile, 2002. Miembro fundador del proyecto de cine itinerante "Elcinevino" entre 1997 y 2003. Ejerció como arquitecto independiente y como jefe de taller del estudio Bresciani Gray Arquitectos. Entre 2006 y 2010 se desempeñó como profesor instructor de la Escuela de Arquitectura Uc en el Área de Tecnologías. En 2010 trabajó dentro del plan de reconstrucción desarrollado por la UC para poblados afectados por el terremoto de Chile. En la actualidad, cursa estudios en el programa de Master of Advanced Studies in Architecture en The University of British Columbia, Canadá.

Hasta inicios del siglo xx, las fachadas de los edificios conformaban su envolvente al mismo tiempo que soportaban las principales cargas estructurales. Sin embargo, las nuevas tecnologías en acero estructural y hormigón reforzado permitieron revolucionarias aplicaciones a la arquitectura. El desarrollo de estructuras más livianas para lograr una mejor iluminación natural condujo al diseño de pilares que liberaron a la envolvente de su función soportante. Consecuentemente, Le Corbusier postuló la independencia total de la fachada con la estructura del edificio, abriendo nuevas posibilidades de diseño que culminaron en la invención del muro cortina como máxima representación de esta condición.

Este tipo de fachadas requiere de una estructura propia dimensionada según el peso de sus componentes, esfuerzos sísmicos y de viento. Los primeros muros cortina fueron desarrollados en acero (FIG.01). Sin embargo, a mediados del siglo XX se masificó el uso del aluminio, que permite extruir perfiles complejos, ampliando las alternativas para fijar los cristales y proteger la envolvente de las condiciones climáticas.

Hoy existe conciencia de que la manufactura de aluminio es una de las labores productivas que presenta mayores índices de "energía incorporada". Este concepto hace referencia a la cantidad de energía no renovable consumida en todas las fases de elaboración del producto. Por ejemplo, son necesarios 32 Mega Joules (MJ) para producir $1 \mathrm{~kg}$ de acero y 1,3 MJ para producir 1 kg de hormigón. En el caso del aluminio, se utilizan 227 MJ $/ \mathrm{kg}^{1}$. La utilización de aluminio

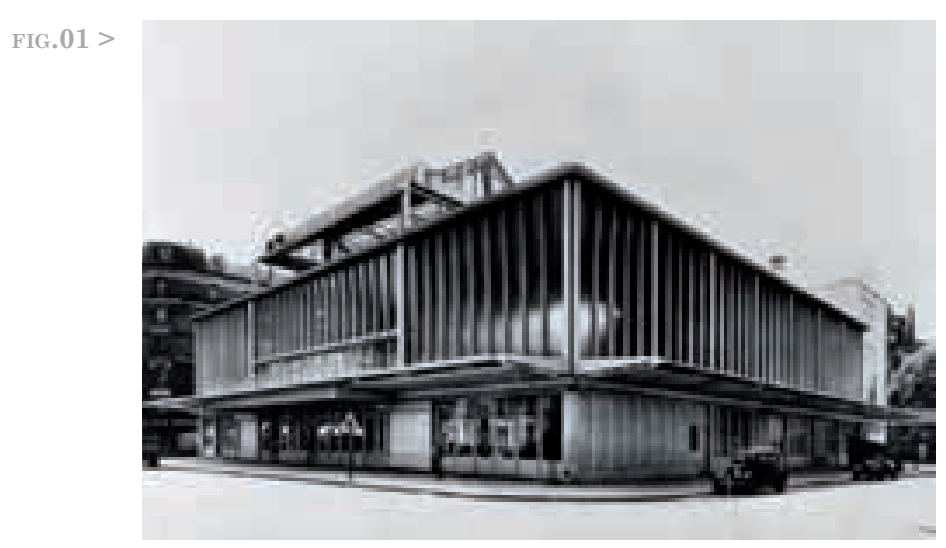

reciclado disminuye considerablemente estos valores, pero en la actualidad no es una solución masificada. Por lo tanto, es necesario buscar soluciones para un diseño de fachadas vidriadas responsable con los recursos y medios disponibles.

La madera es un material de construcción cuya producción, bajo un esquema de forestación sustentable, posee un bajo impacto en el medio ambiente: la energía incorporada en su producción es de 2,5 MJ/kg (FIG.02). En el contexto inmediato, Chile es un país con grandes recursos madereros; el sector forestal representa la segunda actividad económica del país y a partir de los años setenta, la producción ha mostrado un crecimiento, tanto para uso local como para la exportación (Cerda, 2002) (FIG.03).

Existen estudios sobre el desempeño de la madera aplicada a ventanas de pequeñas dimensiones, considerando el consumo de energía estimado de los recintos y emisiones de $\mathrm{CO}_{2}$ en sus etapas de manufacturación, uso, reciclaje y desecho ${ }^{2}$. Los resultados muestran que los valores para las ventanas de madera son significativamente menores que en ventanas de aluminio o de aluminio reciclado (FIG.04).

Sin embargo, para considerar la eficiencia del desempeño de la madera en fachadas vidriadas, se debe tener en cuenta que la pérdida de energía en envolventes se produce principalmente por dos razones: transmisión térmica e infiltraciones.

La transmisión térmica de una fachada depende de las cualidades de su materialidad y aislaciones. Al respecto, el coeficiente de conductividad de la madera representa apenas un $6 \%$ de la del aluminio. Esta propiedad ayuda a mejorar la aislación térmica de la fachada y evitar la formación de puentes térmicos.

1 Valores obtenidos en mayo de 2011 en el sitio web “Measures of Sustainability". http://www.canadianarchitect.com/asf/perspectives_sustainibility/measures_of_sustainablity/measures_of_sustainablity_embodied.htm.

2 El estudio consideró ventanas manufacturadas en España de 1,34 x 1,34 m, instaladas en una pieza estándar según condiciones climáticas de la Península Ibérica (Baldasano, José María et al., 2005). 
FIG.01

Jean Prouvé. Maison du Peuple, Clichy, 1935-39. Muro cortina en estructura de acero.

Fuente: PETERs, Nils. Jean Prouvé 1901-1984 / La dinámica de la creación. Taschen, Colonia, 2006, p.6.

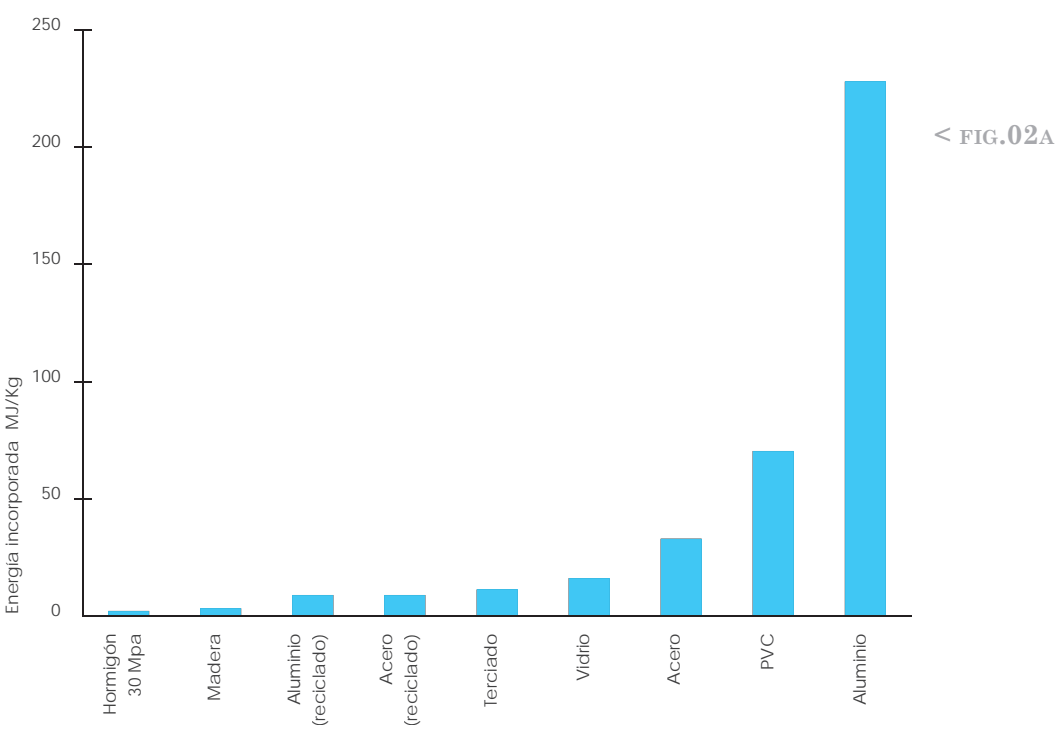

FIG.04A >

Energía incorporada en la producción de materiales de cons trucción. Fuente: elaboración propia a partir de información obtenida en www.canadianarchitect.com.
FIG.03

Producción de madera en Chile. Fuente: elaboración propia

FIG.04 (A Y B)

Consumo de energía y emisiones de $\mathrm{CO}^{2}$ para ciclo de vida de 50 años de ventanas de madera y aluminio. Fuente: elaboración propia

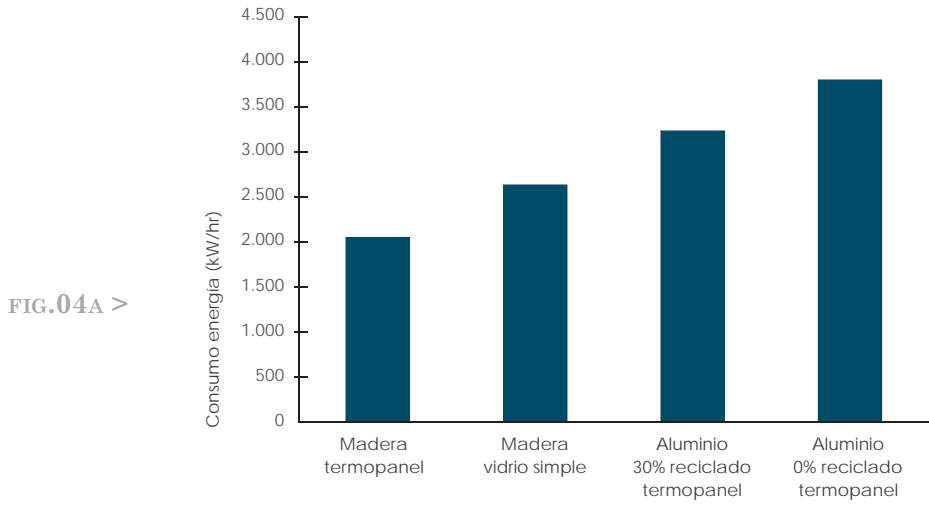

$\begin{array}{lcc}\text { Material } & \begin{array}{c}\text { Energía incoporada } \\ \mathrm{MJ} / \mathrm{Kg}\end{array} \\ \mathrm{MJ} / \mathrm{m}^{3}\end{array}$

$<$ FIG.02B

(n)

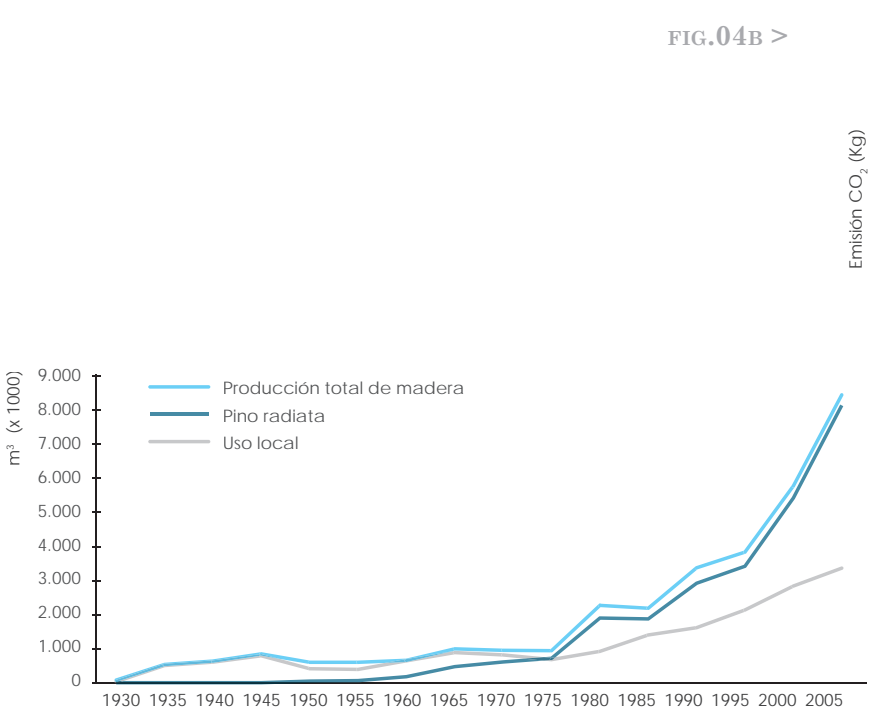

Una parte importante de las filtraciones de energía en la fachada se produce en los encuentros de sus componentes. Desde una perspectiva constructiva, estos encuentros necesitan de una junta de dilatación para coordinar sendos comportamientos de los distintos materiales. A diferencia de lo que ocurre con el coeficiente de dilatación del aluminio $\left(22,2 \times 10^{-6}{ }^{\circ} \mathrm{C}\right)$ y vidrio $\left(5,9-9,0 \times 10^{-6}{ }^{\circ} \mathrm{C}\right)$, los valores del vidrio y madera $\left(3,7-5,4 \times 10^{-6}{ }^{\circ} \mathrm{C}\right)$ son muy similares ${ }^{3}$. Usados en conjunto en el diseño de las fachadas, estos materiales permiten minimizar la dimensión de las juntas de dilatación y optimizar su rendimiento.
La principal desventaja del diseño estructural en madera son sus limitaciones para elaborar elementos refinados o complejos a gran escala en forma económica. Este tipo de producción es compleja, ya que la madera es un compuesto orgánico cuyas irregularidades son propias del material. Existen expectativas de que esta situación cambie a futuro con la incorporación de nuevas tecnologías, como el desarrollo de nuevos compuestos o la masificación de maquinarias Computer Numerical Control (CNC). Sin embargo, actualmente existen alternativas que utilizan medios disponibles en forma innovadora.
Recientemente, en Europa se han producido sistemas de muro cortina donde los mullions son elaborados en madera y las fijaciones para los vidrios en aluminio (FIG.05). La transmitancia térmica de este sistema es hasta un $25 \%$ menor que una solución similar en aluminio ${ }^{4}$.

Otra solución posible consiste en un sistema combinado de una estructura soportante de madera con uniones de acero. Esta alternativa utiliza las propiedades estructurales y térmicas de la madera con las posibilidades de manufacturación personalizada de piezas de acero aplicadas a elementos de unión. Este tipo de conexiones posibilita la prefabricación de las 
piezas, facilita el proceso de montaje y contribuye a la libertad de diseño de la fachada.

Esta lógica es comúnmente utilizada en la obra gruesa de estructuras de madera, pero aún no se ha masificado en sistemas de fachada. Un ejemplo de sus posibilidades puede encontrarse en el proyecto Surrey City Centre (2001) en las cercanías de Vancouver, diseñado por Bing Thom Architects (FIG.06).

Para el diseño de la cubierta y la fachada principal se desarrolló una estructura adaptable a la cambiante geometría del proyecto. En una primera instancia se evaluó una cubierta en acero, pero los arquitectos debieron innovar con una estructura que utiliza piezas desechadas de la manufactura de placas de terciado para ajustarse al presupuesto disponible ${ }^{5}$ (FIG.07).

La estructura de fachada mantiene la misma materialidad. Está compuesta por pilares y mullions horizontales elaborados en Parallel Strand Lumber (PSL) ${ }^{6}$, combinados mediante uniones y tensores de acero (FIG.08). Los pilares constituyen el soporte de cubierta desde la que cuelgan tensores de acero como estructura secundaria. Los mullions horizontales se anclan a los pilares, pero son soportados por los tensores.

Para recibir los esfuerzos horizontales del viento, se incorporaron puntales entre los elementos horizontales y los pilares. El diseño separa los elementos horizontales y verticales de la fachada en distintos planos, acotando los encuentros entre elementos de madera a uniones puntuales. Así, la fachada es percibida como un elemento arquitectónico con espesor (FIG.09).

Pese a la compleja geometría de la fachada, los elementos mantienen un diseño regular y repetitivo que se adapta mediante la configuración de las uniones (FIG.10). Los contratistas de madera y vidrio fueron parte integral del proceso de diseño que incentivó la prefabricación de los elementos de madera para reducir los costos y tiempos de instalación (FIG.11). La utilización de un producto compuesto como PSL facilitó la producción en serie de elementos con diseño y propiedades estructurales homogéneas. El uso de acero forjado incentivó un alto nivel de diseño en las uniones de los distintos componentes, convirtiéndose en elementos con una fuerte carga expresiva que contrastan con la simpleza formal de los elementos de madera (FIG.12).

El diseño final de la fachada resulta en un sistema adaptable por la flexibilidad de sus uniones y fácilmente repetible por su proceso de prefabricación. Como evidencia de la factibilidad y éxito del sistema, los arquitectos utilizaron el mismo diseño de fachada para su proyecto Arena Stage en Washington DC (2004). El único cambio al sistema consistió en reemplazar el vidrio simple por termopanel.

Este ejemplo es solo un caso de exploración en torno a alternativas a las soluciones convencionales utilizando materiales y tecnologías acordes a nuestro tiempo. Para posicionar la madera como material renovable, aplicable a soluciones de fachada, son necesarios diseños innovadores que utilicen los recursos disponibles de forma competitiva en el mercado y responsable con el ambiente. +
FIG.05 (A, B Y C)

Sistema de muro cortina en madera THERM + H-I de Raico

E. 1: 5. Fuente: dibujo elaboración propia / Imágenes de www.raico.de
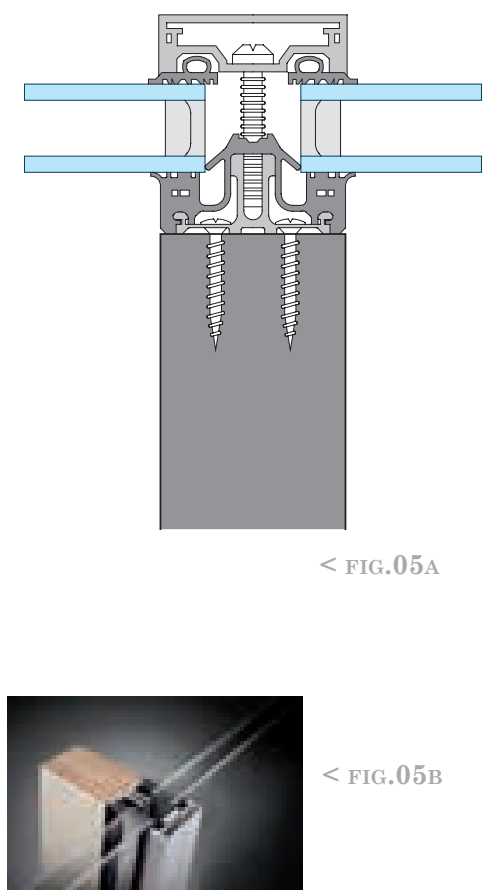

$<$ FIG.05B
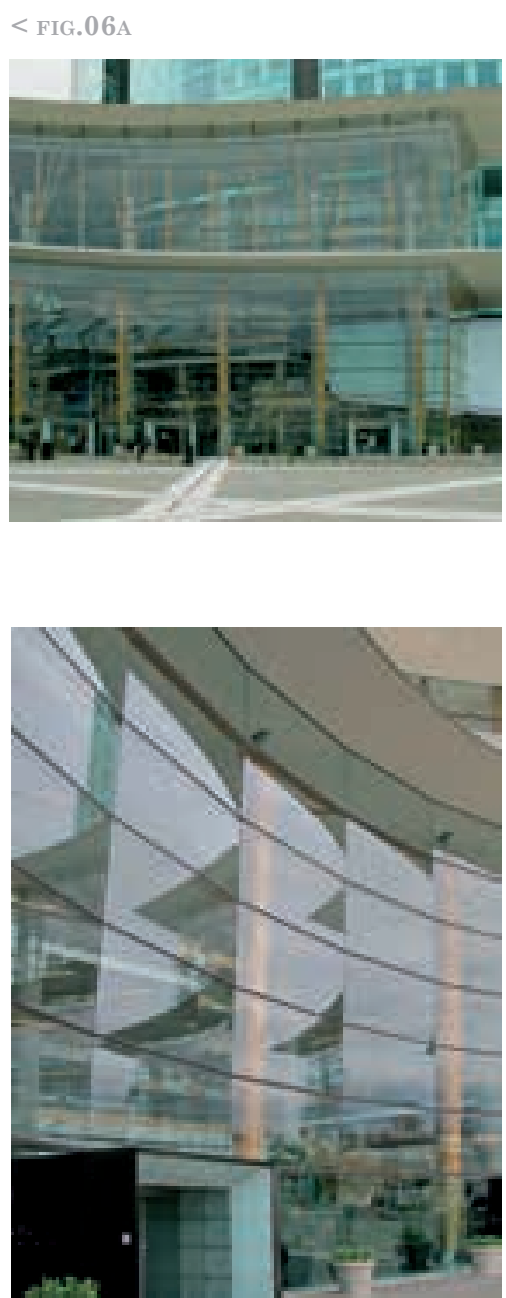

FIG.06 (A Y B)

Bing Thom Architects, Surrey City Centre, 2011. Fuente: fotografías del autor

FIG.07 (А Y B)

Estereométrica compuesta por núcleos de troncos desechados de la manufactura de placas de terciado. Fuente: Archivo de Bing Thom Architects
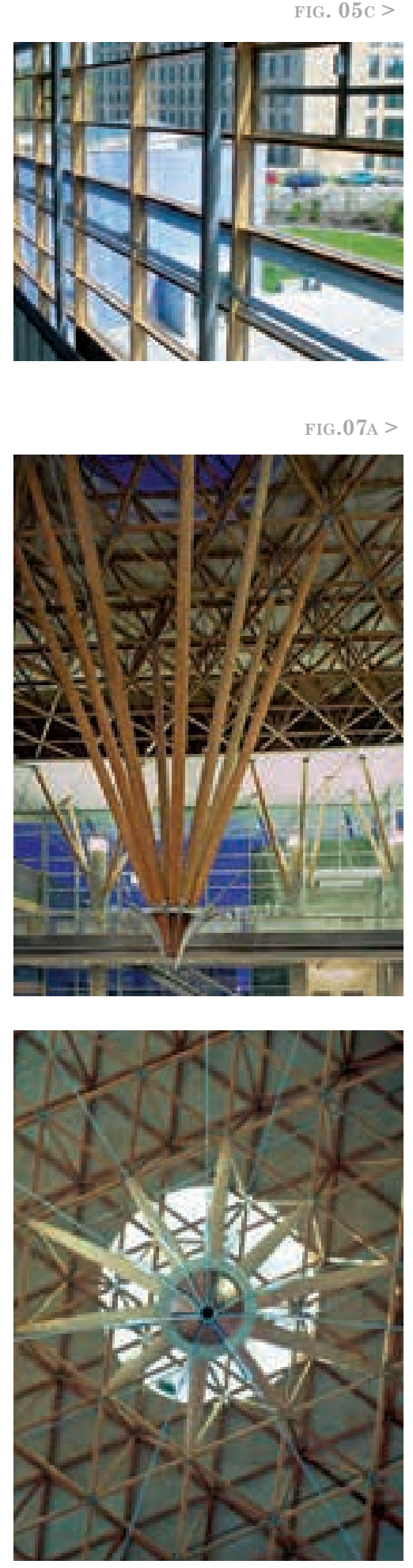

FIG.07B >

FIG.06B >

5 Esta información fue entregada por Francis Yan de Bing Thom Architects, en una entrevista realizada por el autor en abril de 2011.

6 Parallel Strand Lumber (PSL) o "Parallam" es un producto compuesto por astillas de chapas de madera encoladas orientadas en su sección longitudinal. Fue creado a mediados de los años ochenta en Canadá y actualmente es utilizado masivamente en Norteamérica. 
FIG, 08

Esquema de la estructura de fachada, Surrey City Centre E. 1: 1.000

Fuente: elaboración propia

1. Componentes estructurales de mader

2. Componentes vidriados de la fachada

3. Accesos

4. Proyección de cubierta
FIG.09

Esquema constructivo del módulo de fachada, Surrey City Centre

E. 1: 75

Fuente: elaboracion propia

LEYENDA

1. Pilar estructural de madera (PSL)

2. Sistema de anclaje en acero forjado

3. Mullion de madera, muro cortina

4. Vidrio transparente templado $12,7 \mathrm{~mm}$

5. Vidrio transparente laminado $25 \mathrm{~mm}$ (solamente en la base)

nsor de acero

7. Sobrecimiento
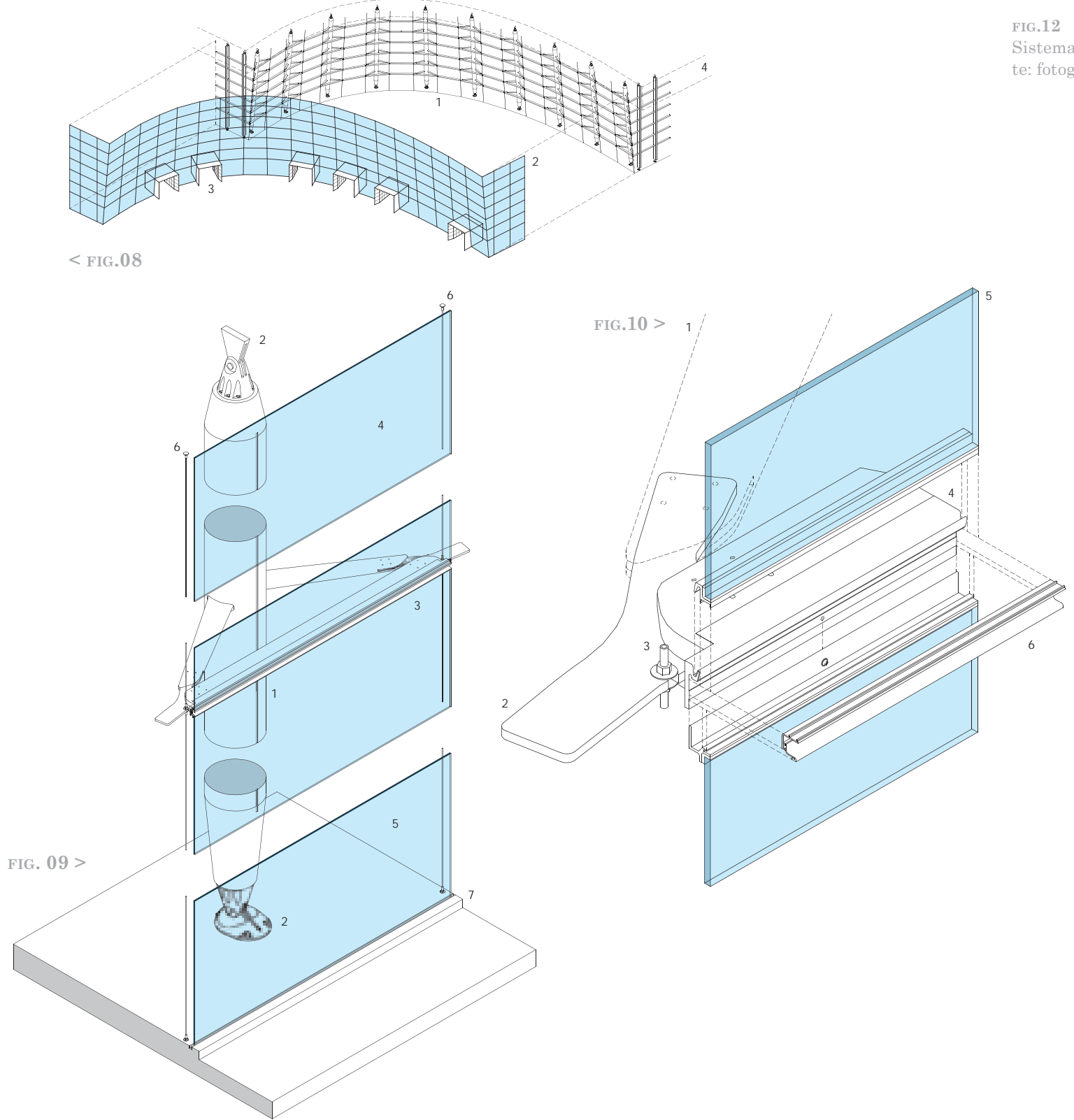

$<$ FIG.11A
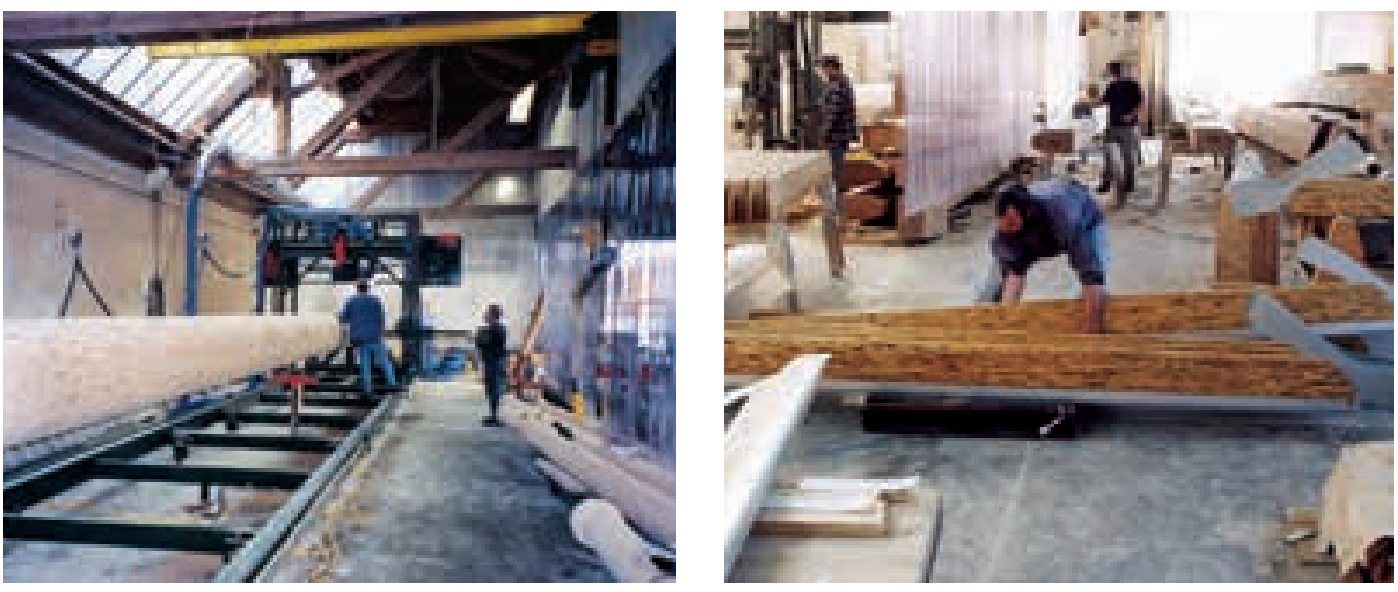

FIG.10

Detalle de la unión entre la estructura y los componente vidriados de la fachada, Surrey City Centre

E. 1: 10. Fuente: elaboracion propia

Leyenda

1. Puntales de madera (PSL)

2. Pieza de unión de acero forjado

.

Mullion de madera con perfiles de aluminio

5. Vidrio transparente templado $12,7 \mathrm{~mm}$

6. Perfil de aluminio para asegurar sello climático

FIG.11 (A, B, C)

Proceso de prefabricación y montaje. Fuente: Archivo de Bing Thom Architects

FIG.12

Sistema de anclaje en acero forjado para pilar de PSL. Fuen te: fotografía del autor

FIG.11C >
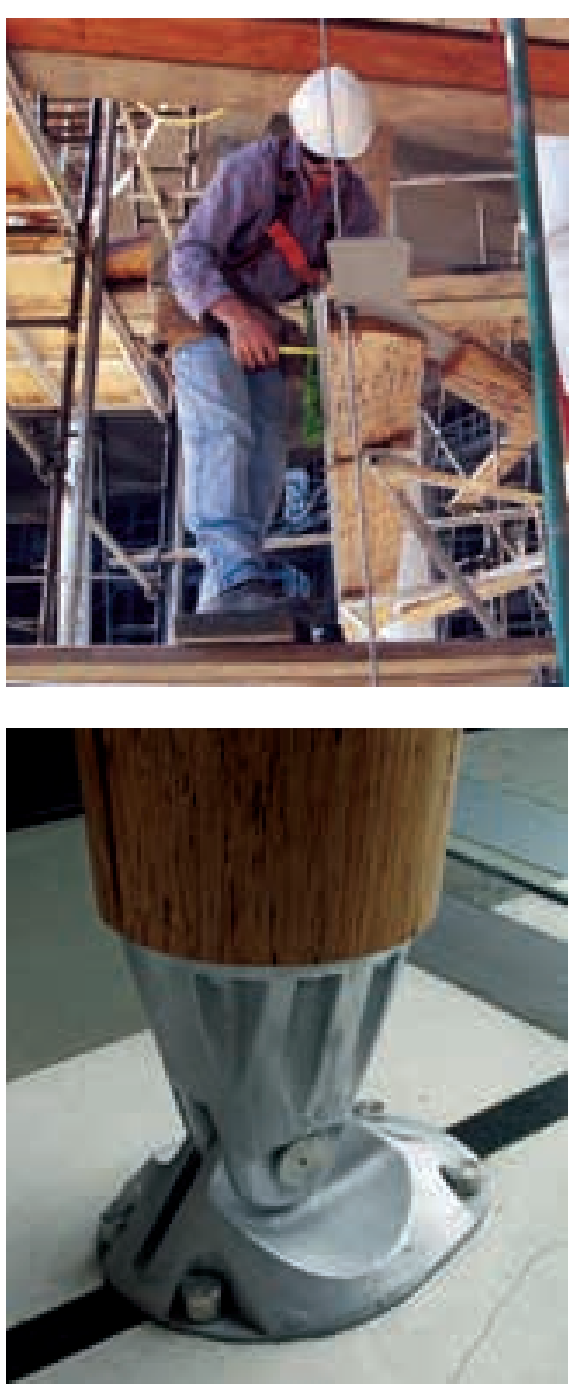

FIG. $12>$

Bibliografía

baldasano, José María, parra, René y Pedro JIMÉNEZ. Estimate of Energy Consumption and $\mathrm{CO}^{2}$

Emission Associated with the Production, Use and Final Disposal of PVD, Aluminium and Wooden Windows. Departament de Projectes d'Enginyeria, Universitat Politécnica de Catalunya, Environmental Modelling Laboratory. Obtenido desde www.pvcinfo.be/bestanden/Baldasano\%20study_windows.pdf. 